1 Habitat complexity and food item size modify the foraging behaviour of a freshwater

2 fish

3

4

5

GREGORY P.D. MURRAY*

6 RICHARD A. STILLMAN

7 J. ROBERT BRITTON

8

9 Department of Life and Environmental Sciences, Faculty of Science and Technology,

10 Bournemouth University, Poole, Dorset, BH12 5BB, United Kingdom

11

12 Running headline: Fish foraging modifications

13

14

15

16

17

18

19

*Corresponding author: Christchurch House, Bournemouth University, Talbot Campus, Fern

20

Barrow, Poole, Dorset, Bournemouth, BH12 5BB, UK

21

Email: gmurray@bournemouth.ac.uk;

22 Tel: +44(0)12026232 ex. 65268; Fax: +44 (0)1202965261

23 Word Count: 8026 
Abstract

The functional response describes the relationship between feeding rate and prey density, and is important ecologically as it describes how the foraging behaviour of an animal changes in response to food availability. The effects of habitat complexity and food item size was experimentally tested here on the foraging parameters and the functional responses of the freshwater fish roach Rutilus rutilus (L.). Habitat complexity was varied through the manipulation of substrate and turbidity, and food item size was varied by using fishmeal pellets, in two sizes. As water turbidity and substrate complexity increased, the reaction distance and consumption rate of the fish significantly decreased. Increased food item size significantly decreased consumption rates but had no influence on any other foraging parameter. Analysis of the interactions between substrate complexity, turbidity and food item size revealed food item size had the greatest influence on consumption rate. Turbidity has the least effect on all the foraging parameters tested. Across all experiments, the functional responses were best described by the Type II response, a relatively consistent finding for $R$. rutilus. These outputs reveal that fish foraging behaviours and functional responses are highly context dependent, varying with environmental parameters and the availability of prey of different sizes. abiotic, Rutilus rutilus 
51 The functional response is the relationship between the feeding rate of a forager and its prey density, and is used to describe and model foraging behaviour (Solomon 1949; Holling 1959; Holling 1966). It is an ecologically important metric as under conditions of limited resource availability, individuals will attempt to maximise their energy intake whilst minimising the costs associated with prey searching and handling (Galarowicz \& Wahl, 2005; Oyugi et al. 2012a,b; Murray et al. 2013). Consequently, measuring how animals respond to variations in food availability helps the understanding of how individuals optimise their foraging behaviour (Werner et al. 1983; Galarowicz \& Wahl, 2005; Murray et al. 2013). This provides knowledge to assist interpretation of the effect of prey availability on consumer condition, growth and fitness (Werner et al. 1983). Moreover, functional responses provide insights into the mechanics of consumer-prey relationships that can have cascading effects through the food web (Koski \& Johnson, 2002). They have considerable ecological applications with, for example, their use as important parameters within individual based models (e.g. Stillman 2008) and as explanatory variables in the success of invasive species (e.g. Bollache et al. 2008; Dick et al. 2013).

Due to how consumers can influence the structure and stability of their prey populations (Alexander et al. 2013), it is ecologically important to distinguish the type of functional response being exhibited (Murray et al. 2013). There are three major function response types: I, II and III (Hassell et al. 1977). Type I describes a linear increase in feeding rate with prey density until it reaches a constant value at saturation (Jeschke et al. 2004) whereas the feeding rate of the Type II response increases at a decreasing rate with prey density until it reaches it maximum value (Holling 1959; Murray et al. 2013). Type II is thus inversely densitydependent and so for the prey population, mortality risks decrease with increasing density 
(Jeschke \& Hohberg, 2008). The Type III response describes a sigmoidal, density-dependent relationship, where an initial increasing risk of prey mortality switches to a decreasing risk of mortality as the prey density increases above a threshold level (Real 1979; Morgan \& Brown, 1996).

Despite their apparent simplicity, it has been established that functional responses are not fixed within specific predator-prey relationships; conversely, under different contexts, foraging and anti-predator behaviours can shift and significantly alter the form of the response (Holling 1959; Alexander et al. 2013). This may involve subtle changes in, for example, the ability of the consumer to detect and respond to the presence of prey items, or may even involve a shift in the functional response type should there be, for example, a substantial increase in the time spent foraging (Abrams 1982). Environmental variables that have been found to influence functional responses, e.g. temperature and light levels (Lipcius \& Hines, 1986; Koski \& Johnson, 2002), and also habitat structure (Alexander et al. 2012). Indeed, habitat structure and complexity have been found to both alter the search ability of the consumer (Savino \& Stein, 1989; Heck \& Crowder, 1991) and the refuge area of their prey (Gotceitas 1990; Warfe \& Barmuta, 2004; Alexander et al. 2012). Prey body size might also be important in determining the values of foraging parameters, given trade-offs between the ease of detection of larger items versus their increased handling time and energetic profitability (Wankowski \& Thorpe 1979; Bean \& Winfield, 1983; Oksanen \& Lundberg, 1995). Nevertheless, there remains a paucity of information on how foraging behaviours are modified between simple and more complex habitats for specific taxonomic groups, such as many benthic feeding freshwater fishes.

Consequently, the aim of this study was to determine how effects of habitat complexity and prey item size, and their interactions, modify the foraging parameters and functional 
response of a model freshwater fish, whose foraging behaviour is generally reliant on visual cues (Hjelm et al. 2003; Bogacka-kapusta 2007). The model species selected was roach Rutilus rutilus (Diehl 1988; Murray et al. 2013), a freshwater fish ubiquitous to many temperate European freshwaters (e.g. Lappalainen et al. 2008) and invasive in others (e.g. Elvira \& Almodovar, 2001; Winfield et al. 2011). Their ecological importance includes their potential for invoking cascading effects on freshwater ecosystems through their high zooplankton grazing rates (e.g. Jeppesen et al. 2010) and thus understanding the contextdependency of their foraging behaviours and functional responses can be ecologically significant. Previous studies have indicated $R$. rutilus can exhibit a Type II (e.g. Johanson \& Persson 1986; Persson 1987; Murray et al. 2013) and Type III (Winkler \& Orellana, 1992) response. These were, however, based on a range of field and experimental approaches, making inter-study comparison of outputs difficult. Thus, here we build on the study of Murray et al. (2013) who used highly controlled experimental conditions to reveal that in a simple environment $R$. rutilus demonstrated a Type II response. We tested the prediction that increases in both habitat complexity and food item size will significantly modify $R$. rutilus foraging behaviour through significantly reduced reaction distance and consumption rate, and increased searching time, handling time and reaction distance, impacting their functional response. 
Ethical note

All animal work was conducted in accordance to national and international guidelines to

127 minimize discomfort to animals. All regulated procedures completed under the Animals

128 (Scientific Procedures) Act 1986 were licensed by the UK Home Office under project licence number PPL 30/2626. The Ethics Review Panel of the School of Applied Sciences of Bournemouth University approved this project licence.

Experimental design overview

133 The experimental design used replicated groups of three $R$. rutilus individuals in experimental arenas, exposed them to different numbers of prey items $(10,25,50,100$ or 150) and captured their foraging behaviours using a two-camera videography system. One camera was positioned horizontally, facing the side of the tank, with the second camera positioned vertically, above the surface of the water. The actual positions and distances moved by the fish were calculated using trigonometry based on footage from both cameras (Murray et al. 2013). The specific details of the experimental arena, video capture, validation of fish movement data, and the use of the Hollings Disc equation for the Type II functional response are detailed in Murray et al. (2013). In summary, there were 12 behavioural arenas (fish aquaria of $0.46 \times 0.31 \times 0.39 \mathrm{~m}$ ) in the experiments that were maintained at $18^{\circ} \mathrm{C}$ on a 12:12 hour light/ dark regime. Three randomly selected roach from a batch of 78 fish (average length $=129 \mathrm{~mm} \pm 2.5 \mathrm{~mm}$; age $1+$ years) were introduced into each arena and allowed to acclimatize to the tanks for 14 days prior to the start of the experiments. pelletized fish-meal ('pellets'). This was due to: (i) the experimental fish were originally 
farmed fish that had been reared on pellets and so were used to consuming them; (ii) cyprinid fish (such as $R$. rutilus) tend to respond well to such pellets in foraging experiments in tanks (e.g. Britton et al. 2012; Oyugi et al. 2012a,b); (iii) as a non-motile 'prey' item that can neither actively select a refuge area, nor display evasive behaviour, measuring the effect of habitat complexity on the consumer would not be confounded by changes in the behaviour of their prey; and (iv) pellets are available in different sizes so food item size could be easily and accurately manipulated. Note that when compared to live prey, the use of pellets precludes the display of more complex foraging behaviours under certain conditions, as prey mobility has been shown to influence feeding rates both negatively, through the confusion 1999). effect (whereby large numbers of evasive prey can reduce attack rates and/or capture efficiencies) (Ioannou et al. 2007; Tosh et al. 2009), or positively, with the movement of prey items increasing predator reaction distance, especially in turbid environments (Utne-Palm

During the experimental period, a single feeding trial, across all the tanks was conducted every other day, with feeding on the day in between comprised of a maintenance ration of approximately $1.5 \%$ body weight. A maintenance ration was used rather than ad libitum to ensure feeding motivation on the experimental days, given that functional responses relate to optimal foraging and therefore behaviour seeking to maximise net energy gain should be promoted. Thus, feeding on experimental days occurred 24 hours after the last exposure to the maintenance ration. Each feeding trial consisted of exposing each tank of fish, in turn, to one randomly selected food density of 10, 25, 50, 100 or 150 pellets per tank (equivalent to 75, 187, 375, 750 and 1125 items $\mathrm{m}^{-2}$ respectively). By discounting a food density previously used in a tank, eventually each of these food item densities was used across all 12 arenas, with the process then being repeated once more (i.e. each food item density was used twice in 
each tank). During the trials, the pellets were introduced to the tank across the entire surface of the water with all pellets sinking through the water column and settling on the base of the tank, with pellets being taken by the fish both as they fell through the water and once they had settled on the bottom of the tank. On the release of the food, the filming of the foraging behaviour commenced for 10 minutes (Oyugi et al. 2012a,b). At the end of this period, all uneaten food was removed immediately using a siphon.

\section{Experimental treatments}

To test the effect of habitat complexity and food item size on the foraging parameters and the functional response, the manipulated parameters were substrate complexity, water turbidity and food item size. The effect of substrate complexity was tested first and then turbidity and food item size.

To test the effect of substrate complexity, the treatments were (1) arenas with no substrate (i.e. simply the glass bottom of the arena) $(n=6)$ and (2) arenas with a layer of dark aquarium gravel (2 to $5 \mathrm{~mm}$ ) of approximately $10 \mathrm{~mm}$ depth on the arena bottom to represent the complex substrate $(n=6)$. Other than the change in substrate, the arenas were identical regarding water turbidly (clear) and food item size (1 mm pellets). These trials were completed separately from the trials of water turbidity and food item size, and used different fish. A table listing the experimental treatments is included in Online Resource 1.

\section{To test the effect of water turbidity and food item size, a two-factor experimental design} was used as it enabled testing of the influence on foraging of both factors and their interactions. These two factors were used together as their interactions will be important in more natural systems where habitats are already complex and their interactions are likely to 
have synergistic effects on a visual forager. Across the 12 arenas, 6 were used with clear water and 6 with water turbidity being increased through addition of a fine powder of bentonite clay to the arena (1g $\pm 0.1 \mathrm{~g}) 5$ minutes prior to the experiments commencing. This was as per Vollset \& Bailey, (2011) who demonstrated the method had no harmful effects to the fish. At the end of each feeding trial, the water turbidity of each arena was quantified through measurement with a turbidity meter (Hanna Instruments, HI 93703 Micro processor, www.hannainst.co.uk), average turbidity in the increased turbidity areas being recorded as equal to $3.41 \pm 0.5 \mathrm{FTU}$, compared to the clear treatments (average $=0.01 \pm 0.0 \mathrm{FTU}$ ), with this difference being significant (ANOVA: $F_{1,528}=110.43, P<0.01$ ). As the clay settled out of solution in approximately six hours, it was then able to be removed by siphoning. The arenas used as clear and turbid water treatments remained constant throughout the experiments.

Across these 12 tanks of varying turbidity, two different sizes of sinking pellets were used: $1 \mathrm{~mm}$ and $2 \mathrm{~mm}$; the numbers released across the trials were as per the substrate experiment (cf. Experimental design). Whilst this meant at a given food density, the biomass of food being introduced would differ between the sizes of pellet, this was justified through functional response analyses generally being based on the consumption rate according to food item density. During each experimental food exposure, the density of food items used was selected randomly for each tank. Once each density had been tested, the trials were later repeated, i.e. each food item density was tested twice in each tank for both pellet sizes. The actual size of pellet used alternated from one experimental food exposure to the next. A table listing the experimental treatments is included in Online Resource 1. 
224 The recorded footage of each feeding trial in both sets of experiments was analysed using a purpose-built event-logger program (Event; Bournemouth University 2012). This allowed frame-by-frame viewing and estimation of the position of objects in three dimensions, enabling parameter estimates of fish foraging behaviour to be measured that formed the basis of the functional response equations (Holling 1959; Murray et al. 2013). These parameters were: (i) swimming speed (s) whilst searching for food, characterised by relatively slow swimming, with frequent changes in body orientation and leading to food item capture; (ii) reaction distance $(d)$, determined as the distance a fish would travel in a straight line directly towards a food item, quickly followed by capture of the food item, often following a change in body orientation towards the food item; and (iii) handling time $(h)$, determined as the time taken to move towards and consume a food item, and then be ready to consume a further food item. Handling time was determined on occasions when food items were captured in rapid succession and when no other behaviour was observed between food item capture. Other parameters recorded, but not used in the functional response equations were: (i) Consumption rate, which was estimated directly, taken as the time between a fish taking its first and fifth food item, and expressed as the number of items consumed per second (Murray et al. 2013). By repeating across the range of food densities, the shape of functional response was able to be described; and (ii) Searching time, recorded as a percentage proportion of the total 242 foraging time that was spent actively searching. This was used to gain insight into the level of risk-taking behaviour displayed by the fish. When perceived risk is reduced, it has been shown that fish will spend a greater proportion of their time searching for food as a compromise between energy intake and potential risks (Milinski \& Heller, 1978; Oksanen \& Lundberg, 1995). 
249 Across the feeding trials in both experiments, there were insufficient data points related to forager parameters collected for each individual fish to enable analyses of their foraging behaviour at that level. Consequently, for the parameters of swimming speed, reaction distance and handling time, separate average parameter values were calculated, based on the arithmetic mean, for each food density and treatment, whilst limiting the number of data points collected for each parameter from any one fish to four, limiting the potential impact of pseudo-replication (Hurlbert 1984). Any further potential impact on the experimental outcomes through familiarisation and learning of optimum feeding behaviour at the experimental food item densities was limited by the use of maintenance rations and time between trials of the same density. Given that the effect of substrate was tested separately with respect to turbidly and food item size, with substrate trials using the same fish population, its effect on the foraging parameters used repeated measures ANOVA. When two factors were being tested (turbidity and food item size experiment) then linear mixed effects models were used, with either food item size or turbidity as a random effect (depending on the test). When comparing the proportion of time spent searching (as a percentage of total experimental time) binomial generalised linear models (GLM) were used.

The foraging behaviour parameters were used to parameterise both a Type I (Jeschke et al. 2002; Jeschke et al. 2004; Murray et al. 2013) and Type II (Holling 1959; Murray et al. 2013) functional response equation, after being tested for density independence (Murray et al 2013). These used the same variables of attack rate (derived from swimming speed and reaction distance) and handling time, together with food item density, differing only in how these parameters were treated. Note that the selection and parameterisation of the functional 
response models, and the estimation of the foraging parameters, are described in more detail in Murray et al. (2013). The Type I model was:

$$
F=\left\{\begin{array}{cl}
a D & \text { if } D \leq \frac{1}{a h} \\
\frac{1}{h} & \text { if } D>\frac{1}{a h}
\end{array}\right.
$$

The Type II model was Holling’s Disc Equation (Holling 1959):

$$
F=\frac{a D}{1+a D h}
$$

Where $F=$ feeding rate (items $\left.\mathrm{s}^{-1}\right), a=$ searching rate (i.e. search area per unit time) $\left(\mathrm{m}^{2} \mathrm{~s}^{-}\right.$ ${ }^{1}$ ), $D=$ food density (items $\mathrm{m}^{-2}$ ) and $h=$ handling time (s) (Holling 1959). In both cases $a$ was defined as:

$$
a=2 d s
$$

Where $s=$ swimming speed $\left(\mathrm{ms}^{-1}\right)$ and $d=$ reaction distance $(\mathrm{m})$.

Thus, the outputs provided the predicted functional response of the fish according to Type I and II equations. These were then compared to the observed functional response i.e. the observed consumption rate data, taken directly from the recorded footage. The best fit between predicted models and observed functional response was then determined by its lower value of the akaike information criterion (AIC) using linear regression models, with each factor (substrate, turbidity and food item size) being tested separately.

Finally, to assess the relative influence of all three factors on the consumption rate and the foraging parameters, as the experimental conditions were the same across both sets of experiments, the data were combined for further testing using linear mixed effects models. To test the relative effects of the factors on each foraging parameter, food density and body length of individual fish were the covariates and experimental arena number was set as a 
random effect (to account for the fact that different experimental arenas were used across the two experiments). Depending on the model, consumption rate and foraging parameters were the dependent variables and were fitted through stepwise removal of non-significant terms according to non-significant $P$ values. All statistics and testing were completed in $\mathrm{R}$ (R version 2.15.1) (R Development Core Team 2012), where reported below, average values include standard deviation $( \pm)$.

\section{Results}

303

\section{Substrate complexity}

The effect of increasing the complexity of the substrate on the foraging behaviours was a significantly decreased reaction distance between the no-substrate (average $=13.2 \pm 5.5 \mathrm{~cm}$ ) and substrate treatment (average $=7.3 \pm 3.9 \mathrm{~cm})\left(\right.$ ANOVA: $\left.\mathrm{F}_{1,18}=6.75, P<0.05\right)$. There was also a significant difference in searching time, with fish in the substrate treatment searching longer (average $=91.0 \pm 3.7 \%$ ) than the no-substrate treatment (average $=28.5 \pm 3.1 \%$; GLM: $\left.F_{1,67}=99.04, P<0.01\right)$. By contrast, there were no significant differences between the treatments for swimming speed and handling time (ANOVA: $F_{1,28}=0.91, P>0.05$ and $F_{1,10}=$ 0.28, $P>0.05$ respectively). See Online Resource 2 for a summary of the behavioural parameter values.

The effect of substrate complexity on the consumption rate of the fish was significant, with reduced rates in the substrate treatment (ANOVA: $F_{1,16}=6.21, P<0.05$; Fig. 1). Comparison between observed functional response and that predicted by the foraging parameters fitted to equations 1, 2 and 3 revealed that a predicted Type II response was the better fit in both substrate and non-substrate treatments (adjusted $\mathrm{R}^{2}=0.94 \mathrm{~F}_{1,3}=48.84, P<$ 
$320 \quad 0.01$ and adjusted $\mathrm{R}^{2}=0.96, \mathrm{~F}_{1,3}=64.86, P<0.01$ respectively) compared to a Type $\mathrm{I}$

321 functional response (adjusted $\mathrm{R}^{2}=0.92, \mathrm{~F}_{1,3}=53.55, P<0.01$; adjusted $\mathrm{R}^{2}=0.94, \mathrm{~F}_{1,3}=$

322 72.52, $P<0.01)$. Similarly, the Type II functional response was a better fit when compared to 323 a simple linear increase (adjusted $\mathrm{R}^{2}=0.91, \mathrm{~F}_{1,3}=68.65, P<0.01$; adjusted $\mathrm{R}^{2}=0.92 \mathrm{~F}_{1,3}=$ 324 77.87, $P<0.01)$. Lastly, the predicted Type II functional response was a better fit than Type I 325 for both the substrate and non-substrate treatment according to AIC (predicted Type II: 326 substrate AIC $=-51.15$; non-substrate AIC $=-44.96$; predicted Type I: substrate AIC = 327 31.42; non-substrate AIC = -14.97).

(Figure 1)

Water turbidity and food item size

When controlling for the effect of food item size, the effect of increased water turbidity was a significant increase in searching time, with fish searching significantly longer (average $=85.0$ $\pm 3.2 \%)$ than in the clear treatments (average $=25.0 \pm 2.9 \%)\left(\mathrm{GLM}: \mathrm{F}_{1,69}=58.21, P<0.01\right)$. Its effect on consumption rate was also significant, with reduced rates in turbid conditions (LMEM: $\mathrm{t}_{74}=-4.37, P<0.01$; Fig. 2). There were, however, no significant differences for swimming speed, reaction distance or handling time between the turbid and clear conditions (LMEM: $\mathrm{t}_{48}=1.43, P=0.13 ; \mathrm{t}_{89}=-2.92, P=0.06 ; \mathrm{t}_{87}=0.149, P=0.88$ respectively). See Online Resource B for a summary of the behavioural parameter values.

When controlling for the effects of turbidity, increasing food item size resulted in a significant reduction in consumption rate (LMEM: $t_{74}=2.51, P=0.02$; Fig. 3). There was, however, no significant effect on searching time, swimming speed, reaction distance or 
handling time (GLM: $\mathrm{F}_{1,69}=2.53, P=0.05 ; \mathrm{LMEM}$ : $\mathrm{t}_{48}=1.22, P=0.18 ; \mathrm{t}_{89}=2.90, P=0.06$ and $t_{87}=-1.57, P=0.11$ respectively).

(Figures 2 and 3)

(Table 1)

350

Factors influencing observed behaviour

The linear mixed effects model (LME) output for all experimental factors combined, revealed 
369

370

371

372

373

374

375

376

377

378

379

380

381

382

383

384

385

386

387

388

389

390

391

392

parameters (Table 2, Online Resource B). The most significant effect on consumption rate was food item size $\left(\mathrm{t}_{223}=8.36, P<0.01\right)$, and for reaction distance and handling time it was substrate complexity $\left(\mathrm{F}_{1,50}=12.3, P<0.01\right)$ and handling time by substrate complexity $\left(\mathrm{t}_{526}=\right.$ 5.19, $P<0.05)$. Within the model, the effects of turbidity on the foraging parameters were not significant.

\section{(Table 2)}

\section{Discussion}

The experiments demonstrated that changes in habitat complexity and food item size had significant consequences for the foraging parameters and functional responses of $R$. rutilus. Thus, aspects of their foraging behaviour were influenced by both their environment and food resources, and this requires consideration in studies that relate to either foraging behaviours and or functional responses. These responses to changing conditions are likely to relate to their foraging being strongly reliant on visual cues (e.g. Aksnes \& Utne, 1997; Diehl 1988; Podolsrky, Uiblein \& Winkler, 1995; Wanzenbock et al. 1996). Regarding the type of functional responses elicited by $R$. rutilus, the best fitting functional response model in each experiment was always Type II. This is a similar outcome to most other studies on $R$. rutilus (e.g. Johanson \& Persson 1986; Persson 1987; Murray et al. 2013). The only exception is Winkler \& Orellana, (1992) where Type III functional response was measured, with this likely to relate to the role of capture probability as a result of evasive behaviour displayed by the live prey. 
Testing of how water turbidity impacted the foraging parameters whilst controlling for the

effect of food item size revealed that consumption rate and reaction distance were reduced as turbidity increased, with this likely to be a result of visual foraging behaviour in roach. It was not considered likely that it was related to changes in olfactory cues as bentonite clay is considered odourless (Vollset \& Bailey, 2011; Zamor \& Grossman, 2007), plus the role of olfaction (compared to visual cues) is limited in roach foraging (Wanzenbock et al. 1996). This outcome is in contrast to findings in three-spined sticklebacks Gasterosteus aculeatus whose reaction distance and attack rate actually increased as turbidity increased (Vollset \& Bailey, 2011). This outcome was related to the altered conditions; both reducing the perceived conspicuousness of the stickleback to potential predators and increasing prey item contrast in the water column, increasing their visibility (Vollset \& Bailey, 2011). Notwithstanding, as the attack rate of G. aculeatus increased their capture success actually decreased, resulting in the consumption rate actually remaining the same and the energetic costs of foraging increasing (Vollset \& Bailey, 2011). The use of pelletized fish meal in our study meant that there was a much more limited role for capture success in shaping the outcome of the foraging, as evasion behaviour was negligible in this experiment and prey refuge was not available, when the gravel substrate was not used. Similarly, Wanzenböck (1995) described the role of prey size selection in altering handling times during foraging by 0+ roach and bleak (Alburnus alburnus); whereby, fish and prey size affected handling time as well as feeding rate, and ultimately, prey profitability (Wankowski \& Thorpe 1979; Buckel \& Stoner 2000). Furthermore, Wanzenböck (1995) showed handling time and prey profitability were seen to vary over the feeding period, as feeding motivation changed. This effect will be limited in our study due to the homogeneity of food item size used within each trial, limiting the potential for prey item selection, altered handling times and profitability. 
The presence of a gravel substrate within the experimental arenas inhibited the majority of the foraging parameters. Within this, and similar, previous experiments (Murray et al. 2013), food pellets were used due to their being a non-motile food item. This was to eliminate the potential confounding effect of the food items actively seeking refuge in more cryptic environments that could result in any shifts in the foraging parameters being due to prey rather than fish behaviour. Indeed, other studies have revealed that functional responses are significantly affected when the refuge area for live prey is increased, as this provides greater opportunities for prey avoidance (e.g. Gotceitas 1990; Warfe \& Barmuta, 2004; Alexander et al. 2012). Nevertheless, in our study, observations on the reduced foraging performance of the $R$. rutilus in the substrate treatment indicated that the reduced consumption rate was largely due to the increased difficulty of the fish being able to detect the pellets once they had settled on the gravel, as the size of pellets allowed a proportion to settle into relatively inaccessible areas (i.e. they provided a 'prey’ refuge). natural conditions should be understood within the context of the relationships described in our study. For example, predation risk, competition and satiation may all affect the rate of feeding (Mills 1982; Persson 1983; Werner et al. 1983; Brabrand \& Faafeng, 1993; Henson

The outcomes of our study highlighted the respective roles of prey item visibility and environmental conditions in determining the foraging behaviours and parameters of a visually foraging fish (Utne-Palm 1999; Sweka \& Hartman, 2003). In natural environments, these dynamic relations are important considerations in habitat selection and optimal foraging, given that foragers will always seek to maximise their energy intake whilst minimising energetic costs and risk of predation (Chick \& McIvor, 1997). As such, the potential role of factors other than prey item density that can influence feeding rate under more complex, \& Hallam, 1995; Elliot 2003; Vahl et al. 2005; Priyadarshana et al. 2006) and, potentially, 
exert a greater influence on feeding rate than that of prey density. Furthermore, given the need to observe feeding behaviour within this study, the 24 hour starvation period used to ensure a high feeding motivation may then be higher than that of fish in the wild, that are less likely to undergo starvation in the same manner (Simpson et al. 1996; Priyadarshana et al. 2006). As such, it can be assumed that it is the short-term functional response of roach that we describe here, rather than the longer term, daily functional response when time is also allocated by individuals to non-feeding activities, and where foraging behaviours must also consider trade-offs with predation risk and competition as described above. In order to more accurately account for the role of feeding motivation in forager behaviours, some roach foraging models have included state-dependent variables that explicitly include the hungerstate of the foragers (Holker et al. 2002; Holker \& Breckling 2005), where hunger-state can dictate behaviours such as habitat selection in association with both resting and feeding activities. Nevertheless, despite these confounding factors, the basic relationships we reveal here between environmental conditions and foraging rate remain valid, even when influenced by further, complex, factors and trade-offs. The results described here will be especially useful in more simple systems, for example in individual-based models (Holker et al. 2002; Railsback \& Harvey 2002).

Frequent changes in the environmental conditions of lowland riverine habitats (where the presence of $R$. rutilus tends to be ubiquitous across their range) are common, in response to prevailing weather conditions or more general shifts in lowland river management. This latter aspect is important given that many lowland river management techniques that are aligned to flood management works substantially modify fish habitats through, for example, removal of instream vegetation that tends to increase turbidity, decrease refugia and increase flow rates (e.g. Allouche \& Gaudin, 2001; Copp 1997; Gregg \& Rose, 1985; Grenouillet \& Pont, 2001). 
467 This suggests that works such as these are likely to affect $R$. rutilus foraging performance, 468 with adult roach switching to less productive filter feeding behaviour in response to 469 environmental challenges. Such changes include increased flow rates and water turbidity

470 (Van Den Berg et al. 2004; Bogacka-kapusta \& Kapusta, 2007; Nurimen et al. 2010) in 471 association with habitat refugia (Garner 1996), and so, the impact of river management on 472 habitat conditions may potentially have substantial implications for fishery and fish 473 population management.

In conclusion, the investigation revealed that foraging parameters and functional responses of $R$. rutilus are modified by changing conditions, with increased complexity tending to 477 decrease aspects of their foraging performance. Increased turbidity, substrate presence and 478 larger food items significantly reduced their consumption rate. In combination, these outcomes suggest that the foraging performance of this species is context-specific; being subject to prevailing conditions and food item availability, and this requires consideration in all relevant applications of their foraging behaviour.

Acknowledgments

484

We thank Calverton fish farm for supplying the fish used in the trials. This study was funded by the Environment Agency and Bournemouth University. 


\section{References}

489 Abrams, P. A., 1982. Functional Responses of Optimal Foragers. The American Naturalist 490 120: 382-390.

491

Aksnes, D. L. \& C. W. Utne, 1997. A revised model of visual range in fish. Sarsia 82: 137492 147.

493

Alexander, M. E., J. T. A. Dick \& N. E. O’Connor, 2013. Trait-mediated indirect interactions 494 in a marine intertidal system as quantified by functional responses. Oikos 122: 1521-1531.

495

496

497

498

499

500

501

502

503

504

505

506

507

508

Alexander, M. E., J. T. A. Dick, N. E., O’Connor, N. R. Haddaway \& K. Farnsworth, 2012. Functional responses of the intertidal amphipod Echinogammarus marinus: effects of prey supply, model selection and habitat complexity. Marine Ecology Progress Series 468: 191202.

Allouche, S. \& P. Gaudin, 2001. Effects of avian predation threat, water flow and cover on growth and habitat use by chub, Leuciscus cephalus, in an experimental stream. Oikos 94: $481-492$.

Bean, C. W. \& I. J. Winfield, 1983. Habitat use and activity patterns of roach (Rutilus rutilus (L.)), rudd (Scardinius erythrophthalmus (L.)), perch (Perca fluviatilis (L.)) and pike (Esox lucius (L.)) in the laboratory: the role of predation threat and structural complexity. Ecology of Freshwater Fish 4: 37-46.

Bogacka-kapusta, E. \& A. Kapusta, 2007. The diet of roach, Rutilus rutilus (L.), and bleak Alburnus alburnus (L.) larvae and fry in the shallow littoral zone of a heated lake. Archives of Polish Fisheries 15: 401-413. 
Bollache, L., J. T. A. Dick, K. D. Farnsworth \& W. I. Montgomery, 2008. Comparison of the functional responses of invasive and native amphipods. Biology Letters 4: 166-9.

Bond, N., D. McMaster, P. Reich, J. R. Thomson \& P. S. Lake, 2010. Modelling the impacts of flow regulation on fish distributions in naturally intermittent lowland streams: an approach for predicting restoration responses. Freshwater Biology 55: 1997-2010.

Britton, J. R., J. Pegg, D. Baker \& C. Williams, 2012. Do lower feeding rates result in reduced growth of a cyprinid fish infected with the Asian tapeworm? Ecology of Freshwater Fish 21: 172-175.

Brabrand, A. \& B. Faafeng, 1993. Habitat shift in roach (Rutilus rutilus) induced by pikeperch (Stizostedion lucioperca) introduction: predation risk versus pelagic behaviour. Oecologia 95: 38-46.

Buckel, J. A. \& A. W. Stoner, 2000. Functional response and switching behavior of youngof-the-year piscivorous bluefish. Journal of Experimental Marine Biology and Ecology 245: 25-41.

Chick, J. H. \& C. C. McIvor, 1997. Habitat selection by three littoral zone fishes: effects of predation pressure, plant density and macrophyte type. Ecology of Freshwater Fish 6: 2735.

Copp, G. H.s 1997. Microhabitat use of fish larvae and 0+ juveniles in a highly regulated section of the River Great Ouse. Regulated Rivers: Research \& Management 13: 267-276.

Dick, J. T. A., K. Gallagher, S. Avlijas, H.C. Clarke, S.E. Lewis, S. Leung \& A. Ricciardi, 2012. Ecological impacts of an invasive predator explained and predicted by comparative functional responses. Biological Invasions 15: 837-846. 
Diehl, S., 1988. Foraging Efficiency of Three Freshwater Fishes: Effects of Structural Complexity and Light. Oikos 53: 207.

Elliott, J. M., 2003. Interspecific interference and the functional response of four species of carnivorous stoneflies. Freshwater Biology 48: 1527-1539.

Elvira, B. \& A. Almodovar, 2001. Freshwater fish introductions in Spain: facts and figures at the beginning of the 21st century. Journal of Fish Biology 59: 323-331.

Event; Bournemouth University (Version 1.0 Software). Available from: http://individualecology. bournemouth.ac.uk/software.html. Last accessed 24 September 2013.

Galarowicz, T. L. \& D. H. Wahl, 2005. Foraging by a young-of-the-year piscivore: the role of predator size, prey type, and density. Canadian Journal of Fisheries and Aquatic Sciences 62: $2330-2342$.

Gotceitas, V, 1990. Variation in plant stem density and its effects on foraging success of juvenile bluegill sunfish. Environmental Biology of Fishes 27: 63-70.

Gregg, W. \& F. Rose, 1985. Influences of aquatic macrophytes on invertebrate community structure, guild structure, and microdistribution in streams. Hydrobiologia 128: 45-56.

Grenouillet, G. \& D. Pont, 2001. Juvenile fishes in macrophyte beds: influence of food resources, habitat structure and body size. Journal of Fish Biology 59: 939-959.

Hassell, M. P., J. H. Lawton \& J. R. Beddington, 1977. Sigmoid functional responses by invertebrate predators and parasitoids. Journal of Animal Ecology 46: 249-262. 
Heck, K. L. Jr. \& L. B. Crowder, 1991. Habitat structure and predator-prey interactions in vegetated aquatic systems. In Bell, S. S., E. D. McCoy \& H. Muchinsky, ed. Habitat Structure: the physical arrangement of objects in space. New York: 281 -299.

Henson, S. M. \& T. G. Hallam, 1995. Optimal feeding via constrained processes. Journal of Theoretical Biology 176: 170-177.

Hjelm, J., G. H. van de Weerd \& F.A. Sibbing, 2003. Functional link between foraging performance, functional morphology, and diet shift in roach (Rutilus rutilus). Canadian Journal of Fisheries and Aquatic Sciences 60: 700-709.

Hölker, F. \& B. Breckling, 2005. A spatiotemporal individual-based fish model to investigate emergent properties at the organismal and the population level. Ecological Modelling 186; 406-426.

Hölker, F., S.S. Haertel, S. Steiner \& T. Mehner, 2002. Effects of piscivore-mediated habitat use on growth, diet and zooplankton consumption of roach: an individual-based modelling approach. Freshwater biology 47: 2345-2358.

Holling, C. S, 1959. Some Characteristics of Simple Types of Predation and Parasitism. The Canadian Entomologist 91: 385-398. 
Holling, C. S, 1966. The functional response of predators to prey density and its role in mimicry and population regulation. Memoirs of the Entomological Society of Canada 48: $5-60$.

Hurlbert, S. H, 1984. Pseudoreplication and the Design of Ecological Field Experiments. Ecological Monographs 54: 187.

Ioannou, C. C., C. R. Tosh, L. Neville \& J. Krause, 2007. The confusion effect from neural networks to reduced predation risk. Behavioral Ecology 19: 126-130.

Jeppesen, E., M. Meerhoff, K. Holmgren, I. González-Bergonzoni, F. Teixeira-de Mello, S. A. J. Declerck, L. Meester, M. Søndergaard, T. L. Lauridsen, R. Bjerring, J. M. CondePorcuna, N. Mazzeo, C. Iglesias, M. Reizenstein, H. J. Malmquist, Z. Liu, D. Balayla \& X. Lazzaro, 2010. Impacts of climate warming on lake fish community structure and potential effects on ecosystem function. Hydrobiologia 646: 73-90.

Jeschke, J. M. \& K. Hohberg, 2008. Predicting and testing functional responses: An example from a tardigrade-nematode system. Basic and Applied Ecology 9: 145-151.

Jeschke, J. M., M. Kopp \& R. Tollrian, 2002. Predator functional Responses: Discriminating between handling and digesting prey. Ecological Monographs 72: 95-112.

Jeschke, J. M., M. Kopp \& R. Tollrian, 2004. Consumer-food systems: why Type I functional responses are exclusive to filter feeders. Biological Reviews 79: 337-349.

Johansson, L. \& L. Persson, 1986. The fish community of temperate eutrophic lakes. In: Riemann, B. \& M. Sondergaard, (eds), Carbon dynamics in eutrophic, temper-ate lakes. Elsevier, Amsterdam: 237-266. 
588 Kasumyan, A. O, 2004. The olfactory system in fish: Structure, function and role in 589 behaviour. Journal of Ichthyology 44: 100-108.

590

591

592

593

594

595

596

597

598

599

600

601

602

603

604

605

606

607

608

Koski, M. L. \& B. M. Johnson, 2002. Functional response of kokanee salmon (Oncorhynchus nerka) to Daphnia at different light levels. Canadian Journal of Fisheries and Aquatic Sciences 716: 707-716.

Lappalainen, J., A. S. Tarkan \& C. Harrod, 2008. A meta-analysis of latitudinal variations in life-history traits of roach, Rutilus rutilus, over its geographical range: linear or non- linear relationships? Freshwater Biology 53: 1491-1501.

Lipcius, R. N. \& A. H. Hines, 1986. Variable functional responses of a marine predator in dissimilar homogeneous microhabitats. Ecology 67: 1361-1371.1

Milinski, M. \& R. Heller, 1978. Influence of a predator on the optimal foraging behaviour of sticklebacks (Gasterosteus aculeatus L.). Nature 275: 642-644.

Mills, N. J., 1982. Satiation and the functional response: a test of a new model. Ecological Entomology 7: 305-315.

Morgan, R. A. \& J. S. Brown, 1996. Using giving-up densities to detect search images. American Naturalist 148: 1059-1074.

Murray, G. P. D., R. A. Stillman, R. E. Gozlan \& J. R. Britton, 2013. Experimental predictions of the functional response of a freshwater fish. Ethology 119: 751-761.

Nurminen, L., Z. Pekcan-Hekim \& J. Horppila, 2010. Feeding efficiency of planktivorous perch Perca fluviatilis and roach Rutilus rutilus in varying turbidity: an individual-based approach. Journal of Fish Biology 76: 1848-55. 
609

610

611

612

613

614

615

616

617

618

619

620

621

622

623

624

625

626

627

628

629

Oksanen, L. \& P. Lundberg, 1995. Optimization of reproductive effort and foraging time in mammals: the influence or resource level and predation risk. Evolutionary Ecology 9: 4546.

Oyugi, D. O., J. Cucherousset, D. J. Baker \& J. R. Britton, 2012a. Effects of temperature on the foraging and growth rate of juvenile common carp, Cyprinus carpio. Journal of Thermal Biology 37: 89-94.

Oyugi, D. O., J. Cucherousset, J. \& J. R. Britton, 2012b. Temperature-dependent feeding interactions between two invasive fishes competing through interference and exploitation. Reviews in Fish Biology \& Fisheries 22: 499-508.

Persson, L, 1983. Food consumption and the significance of detritus and algae to intraspecific competition in roach Rutilus rutilus in a shallow eutrophic lake. Oikos 41: 118-125.

Persson, L, 1987. Effects of habitat and season on competitive interactions between roach (Rutilus rutlius) and perch (Perca fluviatilis). Oecologia 73: 170-177.

Podolsrky, D., F. Uiblein \& H. Winkler, 1995. Visual habitat choice in cyprinid fishes: an experimental approach. Ecology of Freshwater Fish 4: 160-167.

Priyadarshana, T., T. Asaeda \& J. Manatunge, 2006. Hunger-induced foraging behavior of two cyprinid fish: Pseudorasbora parva and Rasbora daniconius. Hydrobiologia 568: $341-352$.

R Development Core Team 2012. R: A language and environment for statistical computing. R Foundation for Statistical Computing, Vienna, Austria. ISBN 3-900051-07-0, URL http://www.R-project.org/. 
630 Real, L. A, 1977. The Kinetics of Functional Response. The American Naturalist

631 111: 289-300.

632 Railsback, S. F. \& B. C. Harvey, 2002. Analysis of habitat-selection rules using an

633 individual-based model. Ecology 83: 1817-1830.

634 Savino, J. F. \& R. A. Stein, 1989. Behavior of fish predators and their prey: habitat choice 635 between open water and dense vegetation. Environmental Biology of Fishes 24: 287-293.

636 Simpson, A. L., N. B. Metcalfe, F. A. Huntingford \& J. E. Thorpe, 1996. Pronounced 637 seasonal diffeences in appetite of atlantic salmon parr, Salmo salar: effects of nutritional 638 state and life-history strategy. Functional Ecology 10: 760-767

639

640

641

642

643

644

645

646

647

648

649

Solomon, M. E, 1949. The Natural Control of Animal Populations. Journal of Animal Ecology 18: 1-35.

Stillman, R. A, 2008. MORPH-An individual-based model to predict the effect of environmental change on foraging animal populations. Ecological Modelling 216: 265276.

Sweka, J. A. \& K. J. Hartman, 2003. Reduction of reactive distance and foraging success in smallmouth bass, Micropterus dolomieu, exposed to elevated turbidity levels. Environmental Biology of Fishes 67: 341-347.

Tosh, C. R., J. Krause \& G. D. Ruxton, 2009. Basic features, conjunctive searches, and the confusion effect in predator - prey interactions. Behavioral Ecology and Sociobiology 63: $473-475$. 
650 Utne-Palm, A. C, 1999. The effect of prey mobility, prey contrast, turbidity and spectral 651 composition on the reaction distance of Gobiusculus flavescens to its planktonic prey. $652 \quad$ Journal of Fish Biology 54: 1244-1258.

653 Vahl, W. K., Van Der Meer, F. J. Weissing, D. V. Van Dullenmen \& T. Piersma, 2005. The 654 mechanisms of interference competition: two experiments on foraging waders. Behavioral $655 \quad$ Ecology 16: 845-855.

656 Van Den Berg, C., J. G. M. Van Den Boogaart, F. A. Sibbing \& J. W. M. Osse, 1994. 657 Zooplankton feeding in common Bream (Abramis brama), White Bream (Blicca 658 Bjoerkna) and Roach (Rutilus rutilus): Experiments, models and energy intake. $659 \quad$ Netherlands Journal of Zoology 44: 15-42.

660 Vollset, K. W. \& K. M. Bailey, 2011. Interplay of individual interactions and turbidity affects 661 the functional response of three-spined sticklebacks Gasterosteus aculeatus. Journal of $662 \quad$ Fish Biology 78: 1954-64.

Wankowski J. W. J. \& J. E. Thorpe, 1979. The role of food particle size in the growth of 664 juvenile Atlantic salmon (Salmo salar L.) Journal of Fish Biology 14: 351-370.

Wanzenböck, J., M. Zaunreiter, C. M. Wahl \& D. L. G. Noakes, 1996. Comparison of 666 behavioural and morphological measures of visual resolution during ontogeny of roach 667 668 (Rutilus rutilus) and yellow perch (Perca flavescens). Canadian journal of Fisheries and Aquatic Sciences 53: 1506-1512.

Wanzenböck J. 1995. Changing handling times during feeding and consequences for prey 670 size selection of 0+ zooplanktivorous fish. Oecologia 104: 372-378. 
671 Warfe, D. M. \& L. A. Barmuta, 2004. Habitat structural complexity mediates the foraging 672 success of multiple predator species. Oecologia: 141, 171-178.

673 Werner, E. E, 1974. The Fish Size, Prey Size, Handling Time Relation in Several Sunfishes 674 and Some Implications. Journal of the Fisheries Research Board of Canada 31: 1531-1536.

675 Werner, E. E., J. F. Gilliam, D. J. Hall \& G. G. Mittelbach, 1983. An Experimental Test of 676 the Effects of Predation Risk on Habitat Use in Fish. Ecology 64: 1540-1548.

677 Winkler, H. \& C. P. Orellana, 1992. Functional responses of five cyprinid species to 678 planktonic prey. Environmental Biology of Fishes 33: 53-62.

679 Zamor, R. G. \& Grossman, 2007. Turbidity Affects Foraging Success of Drift-Feeding 680 Rosyside Dace. Transactions of the American Fisheries Society 136: 167-176.

681

682 
Table 1 Outputs of the linear regression and AIC values, testing the fit of each predicted scores with tests performed separately for each factor.

\begin{tabular}{|c|c|c|c|c|c|c|c|}
\hline Turbidity & Food Item Size & Model & $\mathbf{d f}$ & $\mathbf{R}^{2}$ & $\mathbf{F}$ & $\boldsymbol{P}$ & AIC \\
\hline Turbid & $1 \mathrm{~mm}$ & Type II & 66 & 0.93 & 60.76 & $<0.01$ & -30.26 \\
\hline Turbid & $2 \mathrm{~mm}$ & Type II & 62 & 0.95 & 43.65 & $<0.01$ & -18.68 \\
\hline Turbid & $1 \mathrm{~mm}$ & Type I & 66 & 0.91 & 65.94 & $<0.01$ & -20.65 \\
\hline Turbid & $2 \mathrm{~mm}$ & Type I & 62 & 0.91 & 46.59 & $<0.01$ & -13.89 \\
\hline Turbid & $1 \mathrm{~mm}$ & Linear & 66 & 0.92 & 38.3 & $<0.01$ & -5.65 \\
\hline Turbid & $2 \mathrm{~mm}$ & Linear & 62 & 0.91 & 26.1 & $<0.01$ & -4.45 \\
\hline Clear & $1 \mathrm{~mm}$ & Type II & 59 & 0.93 & 34.71 & $<0.01$ & -8.23 \\
\hline Clear & $2 \mathrm{~mm}$ & Type II & 69 & 0.97 & 66.01 & $<0.01$ & -3.17 \\
\hline Clear & $1 \mathrm{~mm}$ & Type I & 59 & 0.90 & 38.36 & $<0.01$ & 11.47 \\
\hline Clear & $2 \mathrm{~mm}$ & Type I & 69 & 0.92 & 72.6 & $<0.01$ & 13.84 \\
\hline Clear & $1 \mathrm{~mm}$ & Linear & 59 & 0.91 & 49.2 & $<0.01$ & 15.29 \\
\hline Clear & $2 \mathrm{~mm}$ & Linear & 69 & 0.95 & 26.1 & $<0.01$ & 16.38 \\
\hline & & & & & & & \\
\hline
\end{tabular}

686 
Table 2 Outputs of the linear mixed effects models testing the effect of food item size,

689 substrate presence and increased turbidity on consumption rate and foraging parameters.

690 Fixed effects listed by the significance of their effect on each dependent variable. $\mathrm{F}=$ 691 consumption rate; $\mathrm{d}=$ Reaction Distance; $\mathrm{h}=$ Handling Time and $\mathrm{s}=$ Swimming Speed.

\begin{tabular}{|c|c|c|c|c|c|c|c|c|c|c|}
\hline Dependent: & df & $\begin{array}{l}\text { 1st } \\
\text { Factor: }\end{array}$ & $\mathbf{F}$ & $\mathbf{P}$ & $\begin{array}{l}\text { 2nd } \\
\text { Factor: }\end{array}$ & $\mathbf{F}$ & $\mathbf{P}$ & $\begin{array}{l}\text { 3rd } \\
\text { Factor: }\end{array}$ & $\mathbf{F}$ & $\mathbf{P}$ \\
\hline $\mathrm{F}$ & 223 & $\begin{array}{l}\text { Food } \\
\text { Item Size }\end{array}$ & 8.36 & $<0.01$ & Substrate & 0.13 & 0.73 & Turbidity & 2.75 & 0.87 \\
\hline d & 50 & Substrate & 12.32 & 0.01 & $\begin{array}{l}\text { Food } \\
\text { Item Size }\end{array}$ & 0.53 & 0.81 & Turbidity & - & - \\
\hline $\mathrm{h}$ & 526 & Substrate & 5.20 & 0.02 & $\begin{array}{l}\text { Food } \\
\text { Item Size }\end{array}$ & 2.16 & 0.11 & Turbidity & 0.01 & 0.97 \\
\hline S & 1976 & $\begin{array}{l}\text { Food } \\
\text { Item Size }\end{array}$ & 2.55 & 0.07 & Substrate & 0.28 & 0.86 & Turbidity & 1.73 & 0.18 \\
\hline
\end{tabular}

692

693

694 
695

696

697

698

699

700

701

702

703

704

705

706

707

708

709

710

711

712

713

\section{Figure Captions}

Fig.1 Comparison of observed functional responses for the no-substrate (filled squares) versus substrate treatments (clear circles), where the lines represent the modelled Type II functional response from Holling’s Disc Equation parameterised using observed foraging parameters under the no-substrate (solid line), and the substrate treatments (dashed line)

Fig.2 Comparison of observed functional responses for clear water (filled squares) and turbidity treatments (clear circles) using (a) $1 \mathrm{~mm}$ pellets and (b) $2 \mathrm{~mm}$ pellets. The lines represents the modelled Type II functional response from Holling's Disc Equation parameterised using observed foraging parameters, under clear (solid line) and turbid treatments (dashed line)

Fig.3 Comparison of observed functional responses for differences in food item size, where filled squares represent $1 \mathrm{~mm}$ pellets and clear circles $2 \mathrm{~mm}$ pellets and under (a) clear conditions and (b) turbid conditions. The lines represents the modelled Type II functional response from Holling's Disc Equation parameterised using observed foraging parameters, under $1 \mathrm{~mm}$ food item size (solid line) and $2 \mathrm{~mm}$ food item size treatments (dashed line) 